\title{
Tomosyn Negatively Regulates CAPS-Dependent Peptide Release at Caenorhabditis elegans Synapses
}

\author{
Elena 0. Gracheva, Anna 0. Burdina, Denis Touroutine, Martine Berthelot-Grosjean, Hetal Parekh, and \\ Janet E. Richmond \\ Department of Biological Sciences, Science and Engineering Labs, University of Illinois at Chicago, Chicago, Illinois 60607
}

The syntaxin-interacting protein tomosyn is thought to be a key regulator of exocytosis, although its precise mechanism of action has yet to be elucidated. Here we examined the role of tomosyn in peptide secretion in Caenorhabditis elegans tomosyn (tom-1) mutants. Ultrastructural analysis of tom-1 mutants revealed a 50\% reduction in presynaptic dense-core vesicles (DCVs) corresponding to enhanced neuropeptide release. Conversely, overexpression of TOM- 1 led to an accumulation of DCVs. Together, these data provide the first in vivo evidence that TOM-1 negatively regulates DCV exocytosis. In C. elegans, neuropeptide release is promoted by the calciumdependent activator protein for secretion (CAPS) homolog UNC-31. To test for a genetic interaction between tomosyn and CAPS, we generated tom-1;unc-31 double mutants. Loss of TOM-1 suppressed the behavioral, electrophysiological, and DCV ultrastructural phenotypes of unc-31 mutants, indicating that TOM-1 antagonizes UNC-31-dependent DCV release. Because unc-31 mutants exhibit synaptic transmission defects, we postulated that loss of DCV release in these mutants and the subsequent suppression by tom-1 mutants could simply reflect alterations in synaptic activity, rather than direct regulation of DCV release. To distinguish between these two possibilities, we analyzed C. elegans Rim mutants (unc-10), which have a comparable reduction in synaptic transmission to unc-31 mutants, specifically attributed to defects in synaptic vesicle (SV) exocytosis. Based on this analysis, we conclude that the changes in DCV release in tom-1 and unc-31 mutants reflect direct effects of TOM-1 and UNC-31 on DCV exocytosis, rather than altered SV release.

Key words: tomosyn; CAPS; C. elegans; peptidergic transmission; neuromuscular junction; UNC-31; TOM-1

\section{Introduction}

The calcium-dependent fusion of secretory vesicles requires soluble $N$-ethylmaleimide-sensitive factor attachment protein receptor (SNARE) complex assembly between the vesicle R-SNARE synaptobrevin and plasma membrane-associated Q-SNAREs syntaxin and synaptosomal-associated protein-25 (SNAP-25) (Sollner et al., 1993; Broadie et al., 1995; Hanson et al., 1997; Lonart and Sudhof, 2000; Chen and Scheller, 2001). Given the critical importance of SNARE complex formation, molecules that regulate this process are likely to be important modulators of neurosecretion. Tomosyn is a pan-neuronal syntaxin-binding protein that has two conserved domains: a C-terminal SNARE motif that competes with synaptobrevin for assembly into tertiary complexes with syntaxin and SNAP-25 (Fujita et al., 1998; Yokoyama et al., 1999; Hatsuzawa et al., 2003; Pobbati et al., 2004) and an N-terminal WD40 repeat domain, predicted to form two $\beta$ propellers that may bind SNAP-25 (Hat-

Received May 22, 2007; revised Aug. 5, 2007; accepted Aug. 8, 2007.

This work was supported by National Institutes of Health Grants R01 MH073156-01A2 and R01 NS041477. We thank Dr. Brian Ackley for constructive criticism of this manuscript; Dr. Kenneth Miller for providing the UNC-31 antibody; Dr. Joshua Kaplan for KP\#1383 punc-129::nlp-21::Venus; Linda Juarez, Kristina Jarosius (Research Resources Center Electron Microscopy facility, University of Illinois at Chicago, Chicago, IL), and Jay Campbell (Laboratory for Optical and Computational Instrumentation, University of Wisconsin-Madison, Madison, WI) for technical assistance with the high-pressure freeze EM; and The Caenorhabditis Genetics Center for providing strains.

Correspondence should be addressed to Dr. Janet E. Richmond, Department of Biological Sciences, Science and Engineering Labs, 840 West Taylor Street, University of Illinois at Chicago, Chicago, IL 60607. E-mail: jer@uic.edu. DOI:10.1523/JNEUROSCI.2339-07.2007

Copyright $\odot 2007$ Society for Neuroscience ～0270-6474/07/2710176-09\$15.00/0 tendorf et al., 2007). In an effort to determine the function of tomosyn, several groups have demonstrated that tomosyn overexpression inhibits synaptic vesicle (SV) exocytosis in neurons (Baba et al., 2005; Gracheva et al., 2006) and dense-core vesicle (DCV) release in endocrine cells (Fujita et al., 1998; Hatsuzawa et al., 2003; Yizhar et al., 2004; Zhang et al., 2006). On the basis of these data, tomosyn has been proposed to act as a negative regulator of both types of vesicles. However, RNA interference (RNAi) experiments in cultured cells have produced conflicting results (Baba et al., 2005; Cheviet et al., 2006; Zhang et al., 2006) leading to speculation that tomosyn may have permissive as well as inhibitory functions.

We previously characterized the SV transmission phenotype of mutants in the single Caenorhabditis elegans tomosyn gene tom-1 (Dybbs et al., 2005). Both pharmacological assays and direct measurements of synaptic transmission demonstrate that SV priming and, consequently, neurotransmitter release are enhanced in tom-1 mutants (Gracheva et al., 2006; McEwen et al., 2006). Furthermore, tom-1 mutants partially suppress the complete loss of priming associated with unc-13 mutants. These antagonistic actions of UNC-13 and TOM-1 may arise from their mutual interaction with syntaxin: UNC-13/syntaxin interaction promoting SNARE complex assembly (Richmond et al., 2001; Madison et al., 2005; Stevens et al., 2005) and TOM-1/syntaxin interaction inhibiting SNARE complex assembly (Hatsuzawa et al., 2003).

The fact that release characteristics of SVs and DCVs differ substantially (Bruns and Jahn, 1995) suggests that the molecular 
machinery regulating these events may also be distinct. For example, although overexpression of Munc13 in chromaffin cells is capable of enhancing the number of primed DCVs (Ashery et al., 2000), DCV secretion is normal in Munc13-1 mutants (Stevens et al., 2005), suggesting other factors render DCVs fusion competent. Calcium-dependent activator for protein secretion (CAPS) is a leading contender for this DCV priming factor (Ann et al., 1997; Berwin et al., 1998; Renden et al., 2001). Analysis of CAPS mutants in both Drosophila (dCAPS) and C. elegans (unc31) support a permissive role for this conserved protein in peptide secretion (Sieburth et al., 2006). Given that there may be distinct priming mechanisms for SVs and DCVs, in this study we addressed whether TOM-1 has a role in regulating DCV release, and whether it genetically interacts with C. elegans UNC-31.

\section{Materials and Methods}

Genetics

Nematodes were maintained on agar plates seeded with OP50 bacteria. Strains used were N2 Bristol, VC223 tom-1(ok285), KP3293 tom1(nu468), unc-104(e1265), SY1181 tom-1(nu468);jaIs1052 (integrated Punc-17::TOM1A), unc-31(e928), SY1206 tom-1(ok285);unc31(e928), unc-10(md1117), SY1006 unc-10(md1117);tom-1(ok285), KP\#1383 punc-129::nlp-21::Venus, unc-31(e928), and SY1226 KP\#1383;tom-1(ok285).

\section{Electron microscopy}

High-pressure freezing and freeze substitution. Young adult hermaphrodites for each strain were prepared for high-pressure freezing as described previously (Rostaing et al., 2004). Briefly, 10-15 animals were loaded in a specimen chamber filled with Escherichia coli and immobilized by high-pressure freezing at $-180^{\circ} \mathrm{C}$ under high pressure in a BalTec HPM010 and moved to liquid nitrogen.

Freeze substitution was performed in a Reichart AFS machine (Leica, Oberkochen, Germany) as described previously for morphological analysis, using tannic acid $(0.1 \%)$ and $0.5 \%$ gluteraldehyde fixative introduced over $4 \mathrm{~d}$, followed by $2 \%$ osmium (Weimer and Richmond, 2005). Fixed specimens were then embedded in Araldite 502 over a $48 \mathrm{~h}$ period at $60^{\circ} \mathrm{C}$. For immunoEM analysis, animals were fixed with $0.1 \%$ potassium permanganate for $72 \mathrm{~h}$ and embedded in Lowicryl for $60 \mathrm{~h}$ under UV light.

Serial sections were cut at a thickness of $40-50 \mathrm{~nm}$, collected on formvar-covered, carbon-coated copper grids (EMS, FCF2010-Cu), and counterstained in 2 or $2.5 \%$ aqueous uranyl acetate for $4 \mathrm{~min}$, followed by Reynolds lead citrate for 2 min. Images were obtained on a Jeol (Tokyo, Japan) JEM-1220 transmission electron microscope operating at 80 $\mathrm{kV}$. Micrographs were collected using a Gatan (Pleasanton, CA) digital camera at a magnification of $60 \times$ and $100 \times$.

Morphometric analysis of ventral nerve cord serial sections was scored blind. Images were quantified using NIH Image software. A synapse was defined as a set of serial sections containing a presynaptic specialization and two flanking sections from both sides without presynaptic specialization.

Post-embedding immunogold labeling. Thin $(\sim 50 \mathrm{~nm})$ sections from Lowicryl-embedded samples were collected as ribbons on formvarcoated nickel slot grids and immunogold labeled in a similar manner to that described previously (Rostaing et al., 2004). The UNC-31 primary antibody was used at a dilution of 1:50, and the anti-goat $10 \mathrm{~nm}$ gold bead-conjugated antibody was diluted 1:150, the TOM-1A primary antibody was used at a dilution of 1:300, and the secondary anti-rabbit 15 $\mathrm{nm}$ gold bead-conjugated antibody was diluted 1:100.

Coelomocyte NLP-21::YFP image analysis. This recently developed peptide secretion assay (Sieburth et al., 2006) involves immobilizing wild-type, tom-1(ok285), and unc-31(e928) mutant young adult worms expressing NLP-21::YFP (KP\#3183) under the cholinergic promoter punc-129 and imaging the posterior coelomocyte. Image stacks were collected on an Olympus (Tokyo, Japan) FV500 confocal microscope and quantified using NIH Image. Fluorescent values for each coelomocyte were subsequently normalized to the wild-type average.
Electrophysiology. Electrophysiological methods were as described previously (Richmond et al., 1999; Gracheva et al., 2006). Briefly, animals were immobilized with cyanoacrylic glue, and a lateral cuticle incision was made to expose the ventral medial body-wall muscles. Muscle recordings were made in the whole-cell voltage-clamp configuration (holding potential, $-60 \mathrm{mV}$ ) using an EPC-10 patch-clamp amplifier and digitized at $2.9 \mathrm{kHz}$. The extracellular solution consisted of the following (in mM): $150 \mathrm{NaCl}, 5 \mathrm{KCl}, 5 \mathrm{CaCl}_{2}, 4 \mathrm{MgCl}_{2}, 10$ glucose, 5 sucrose, and 15 HEPES, pH $7.3(\sim 340 \mathrm{mOsm})$. The patch pipette was filled with the following (in $\mathrm{mm}$ ): $120 \mathrm{KCl}, 20 \mathrm{KOH}, 4 \mathrm{MgCl}_{2}, 5$ $\mathrm{N}$-tris[hydroxymethyl] methyl-2-aminoethane-sulfonic acid, 0.25 $\mathrm{CaCl}_{2}, 4 \mathrm{Na}_{2} \mathrm{ATP}, 36$ sucrose, and 5 EGTA, pH 7.2 ( $\left.\sim 315 \mathrm{mOsm}\right)$. Hyperosmotic responses acquired by pressure ejecting extracellular saline adjusted to $\sim 840 \mathrm{mOsm}$ with sucrose for $1.5 \mathrm{~s}$ were measured as the total charge integral for minis during the hyperosmotic response minus the preceding $1.5 \mathrm{~s}$ of control minis. Data were acquired using Pulse software (HEKA Elektronik, Lambrecht/Pfalz, Germany) run on a Dell computer. Subsequent analysis and graphing was performed using Pulsefit (HEKA Elektronik), Mini analysis (Synaptsoft, Decatur, GA), and Igor Pro (Wavemetrics, Lake Oswego, OR).

Immunohistochemistry. Immunohistochemistry was performed as described previously (Gracheva et al., 2006) using a modified method of the whole-worm fixation (Charlie et al., 2006). Briefly, worms were frozen on dry ice between two glass coverslides, which were then split apart. Frozen animals were fixed in $4 \%$ slushy formaldehyde in PBS. After fixation, animals were collected by column filtration (Pierce, Rockford, IL). Antibodies against UNC-31 were used at a final dilution of 1:200 in PBS and $0.5 \%$ Triton X-100 with 3\% BSA. Anti-goat tetramethylrhodamine isothiocyanate-conjugated secondary antibody (Jackson ImmunoResearch, West Grove, PA) was used at a 1:500 dilution for $4 \mathrm{~h}$ at $4^{\circ} \mathrm{C}$. Images were obtained with a $60 \times$ objective on an Olympus Optical FV500 laser-scanning confocal microscope.

\section{Behavioral assays}

Locomotion assays were performed on OP50 seeded plates. Ten worms of each genotype tested were individually plated and allowed a $1 \mathrm{~min}$ settling period before the trial was performed. Basal locomotion rates and reversal responses to eyelash head-taps were video recorded and quantified as body bends per unit time.

\section{Results}

\section{TOM-1 regulates DCV abundance}

Previous studies have established that neuropeptide release in $C$. elegans is dependent on UNC-31(CAPS) (Sieburth et al., 2006). Because both UNC-31 and TOM-1 are highly expressed at the electrophysiologically accessible neuromuscular junctions (NMJs) of C. elegans (Charlie et al., 2006; McEwen et al., 2006), we focused our analysis on these synapses. To assess whether tomosyn regulates peptide release at the NMJs, we compared the number of DCVs in wild-type and tom-1 loss-of-function mutants. Worms were prepared for ultrastructural analysis using high-pressure freeze and freeze-substitution fixation techniques, serially sectioned, and imaged along the ventral nerve cord where NMJs are abundant (Fig. $1 A$ ). The analysis of synaptic profiles in these EM sections revealed a significant reduction in the number of DCVs at NMJs in two tom-1 alleles, relative to the number at NMJs of wild-type worms. Specifically, we observed 50\% fewer DCVs in tom-1 (ok285) and $~ 60 \%$ fewer DCVs in tom-1(nu468) than in wild-type synaptic profiles (Fig. $1 B$, Table 1). These results suggest that in the absence of TOM-1, DCV release is enhanced, leading to a depletion of the DCV pool at the NMJs of tom-1 mutants.

If loss of TOM-1 leads to increased DCV fusion, we would expect overexpression of TOM-1 to have the opposite effect, inhibiting DCV release. We therefore examined the number of DCVs in tom-1(nu468) mutants overexpressing the TOM-1A isoform ( jaIs1052) in cholinergic motor neurons (Gracheva et 
A
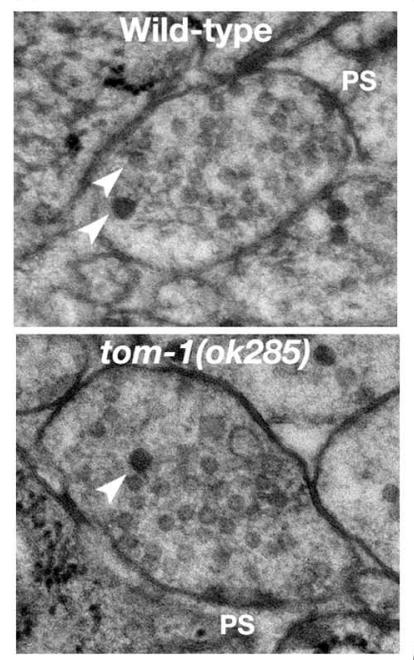

tom $1(1 \mathrm{nut} 68)$
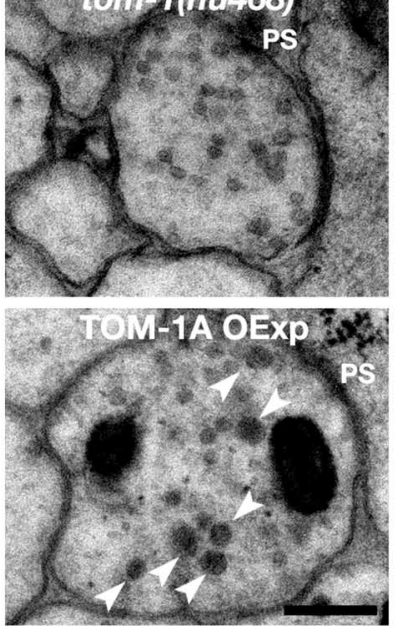

B
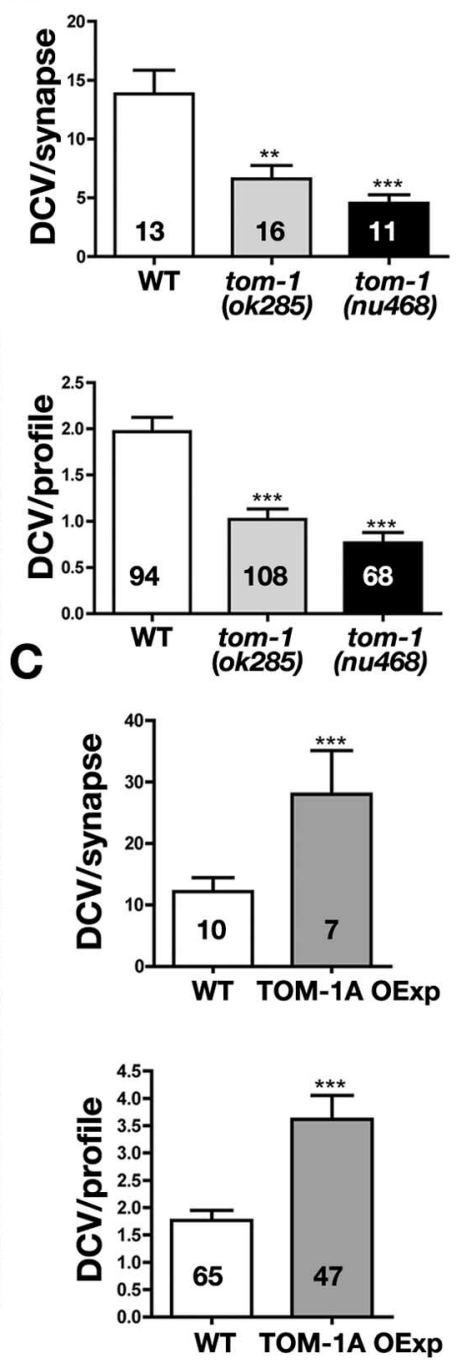

Figure 1. Ultrastructural evidence that tomosyn negatively regulates the release of DCVs from C. elegans NMJs. $A$, Representative micrographs containing NMJs from the ventral nerve cord of wild-type, tom-1(ok285), tom-1(nu468), and TOM-1A-overexpressing worms ( jals1052). The electron-dense presynaptic specialization (PS) of the NMJ in each image is indicated. Arrowheads identify DCVs. Scale bar, $200 \mathrm{~nm}$. $\boldsymbol{B}$, The number of DCVs per synapse [defined as consecutive synaptic profiles with a presynaptic specialization (PS) and two flanking sections on either side of the PS containing profiles] was counted for each NMJ in wild type, tom-1(ok285), and tom-1(nu468). The top graph plots the average number of DCV s per synapse, and the bottom graph plots the average number of DCVs per profile for each strain. The significance values (asterisks) above the bars are relative to the wild type, and numbers in the bars represent the sample size ( $n$ ). C, Graphs of average DCV per synapse (top) and average DCV per profile (bottom) calculated for TOM-1A-overexpressing cholinergic NMJs relative to wild-type cholinergic synapses. Data in all figures are expressed as mean \pm SEM. TOM-1A OExp, TOM-1Aoverexpressing; WT, Wild type.

al., 2006). The number of DCVs was significantly increased in TOM-1A-overexpressing neurons, rising from $1.77 \pm 0.185$ $\mathrm{DCV} /$ profile $(n=65$ profiles; $12.2 \pm 2.2 \mathrm{DCV} /$ synapse; $n=10$ synapses) in wild-type cholinergic synapses to $3.62 \pm 0.44 \mathrm{DCV} /$ profile ( $n=47$ profiles; $28 \pm 7.1 \mathrm{DCV} /$ synapse; $n=7$ synapses) in jaIs1052 cholinergic synapses (Fig. $1 A, C$ ). Together, these data are consistent with the hypothesis that DCV fusion is negatively regulated by TOM-1 at C. elegans NMJs. However, based on EM alone, we cannot rule out the possibility that TOM-1 is required for DCV biogenesis.

To distinguish between these two possible roles for TOM-1

(i.e., DCV biogenesis or regulation of DCV secretion), we used a recently developed imaging assay of neuropeptide release, in which the level of secreted yellow fluorescent protein (YFP)tagged neuropeptide NLP-21 can be measured (Sieburth et al., 2006). This assay takes advantage of the fact that once secreted, NLP-21::YPF is taken up by scavenger cells known as coelomocytes, the fluorescence intensity of these discrete structures providing an indirect readout of peptide secretion (Sieburth et al., 2006) (Fig. 2A). We generated tom-1 mutants expressing NLP-21::YFP in the cholinergic motor neurons and compared the coelomocyte fluorescence levels to wild-type worms with the same NLP-21::YFP transgene. The coelomocyte NLP-21::YFP signal was $50 \%$ brighter in tom-1 mutants compared with the wild type (Fig. $2 B, C$ ), indicating that peptide release from cholinergic motor neurons in tom-1 mutants is enhanced. These data indicate that the loss of DCVs observed in tom-1 mutants is the result of enhanced peptide release rather than reduced DCV biogenesis.

\section{UNC-31 is required for DCV release}

Mammalian tomosyn has been shown to compete with synaptobrevin in the assembly of tertiary complexes with syntaxin and SNAP-25 (Hatsuzawa et al., 2003), leading to the hypothesis that tomosyn inhibits vesicle exocytosis by restricting the assembly of synaptobrevin SNARE complexes required for fusion. Consistent with this model, antagonistic roles have been demonstrated for $C$. elegans TOM-1 and the SV priming factor, UNC-13 (Richmond et al., 1999; Gracheva et al., 2006; McEwen et al., 2006). UNC-31, the C. elegans homolog of vertebrate CAPS, may play a similar role to UNC-13 in the promotion of DCV release (Sieburth et al., 2006). To test the functional relationship between TOM-1 and UNC-31, we first analyzed the phenotype of unc-31 mutants.

Although mammalian CAPS is known to be a cytosolic protein (Walent et al., 1992), UNC-31 antibody staining in C. elegans is observed as discrete puncta that colocalize with presynaptic markers at NMJs and central synapses (Charlie et al., 2006). Because mammalian CAPS has been shown to bind to DCVs (Berwin et al., 1998) via a conserved C-terminal domain (Grishanin et al., 2002), we postulated that the synaptic localization of UNC-31 might reflect its interaction with DCVs at C. elegans NMJs. To test this prediction, we examined the distribution of UNC-31 at the immunoEM level. Individual gold beads bound to UNC-31 were found to be associated with DCVs at NMJ terminals (Fig. $3 A$ ), and the overall bead distribution corresponded with that of both DCVs and TOM-1 immunoEM (Fig. $3 B, C$ ). As observed in previous studies, DCVs are not as closely associated with the presynaptic specialization as SVs (Weimer et al., 2006). These data suggest that UNC-31 (and TOM-1) associate with peptidecontaining DCVs in C. elegans. This was also supported by the observation that UNC-31 immunofluorescence accumulates in neuronal cell bodies in mutants of C. elegans kinesin (UNC-104), a microtubule motor protein required to transport peptides to synaptic terminals (Zahn et al., 2004; Speese et al., 2007) (Fig. $3 D$ ). These data suggest that UNC-31 targeting to DCVs may be similar to its mammalian ortholog, CAPS (Berwin et al., 1998).

Several studies have shown that CAPS is an essential cytosolic component required to reconstitute calcium-dependent peptide release, acting at a late step in DCV fusion (Hay and Martin, 1992; Walent et al., 1992; Ann et al., 1997). In C. elegans, unc-31 mutants have been shown to accumulate fluorescently tagged peptides in cholinergic NMJs with concomitant reductions in the levels of released peptides sequestered in coelomocytes (Sieburth et al., 2006; Speese et al., 2007), an observation that we confirmed 
Table 1. Ultrastructural and electrophysiological data summary

\begin{tabular}{|c|c|c|c|c|}
\hline Genotype & DCV/profile & DCV/synapse & Evoked amplitude(pA) & Evoked charge(pc) \\
\hline Wild type & $1.97 \pm 0.16(n=94)$ & $13.85 \pm 2.02(n=13)$ & $2305 \pm 998(n=49)$ & $17.1 \pm 0.83(n=49)$ \\
\hline tom-1(nu468) & $0.77 \pm 0.11(n=68)$ & $4.45 \pm 0.72(n=11)$ & $2560 \pm 283(n=11)$ & $42.6 \pm 5.6(n=8)$ \\
\hline tom-1(ok285) & $1.02 \pm 0.12(n=108)$ & $6.63 \pm 1.14(n=16)$ & $2426 \pm 107(n=25)$ & $36.7 \pm 3.0(n=25)$ \\
\hline TOM-1A (jals1052) & $3.62 \pm 0.44(n=47)$ & $28 \pm 7.1(n=7)$ & Gracheva et al. (2006) & Gracheva et al. (2006) \\
\hline unc-31(e928) & $5.42 \pm 0.36(n=65)$ & $37.6 \pm 4.44(n=9)$ & $840 \pm 126(n=10)$ & $3.8 \pm 0.49(n=10)$ \\
\hline tom-1;unc-31 & $3.2 \pm 0.2(n=69)$ & $21.6 \pm 2.12(n=10)$ & $1566 \pm 179(n=16)$ & $12.75 \pm 1.82(n=16)$ \\
\hline unc-10(md1117) & $2.66 \pm 0.24(n=86)$ & $18.4 \pm 3.63(n=9)$ & $846 \pm 77.3(n=5)$ & $\begin{array}{l}4.55 \pm 0.6(n=5) \\
10.6 \pm 1.7(n=6)\end{array}$ \\
\hline tom-1;unc-10 & $0.98 \pm 0.17(n=41)$ & $6.67 \pm 1.78(n=6)$ & $1544 \pm 193.3(n=6)$ & \\
\hline
\end{tabular}

A

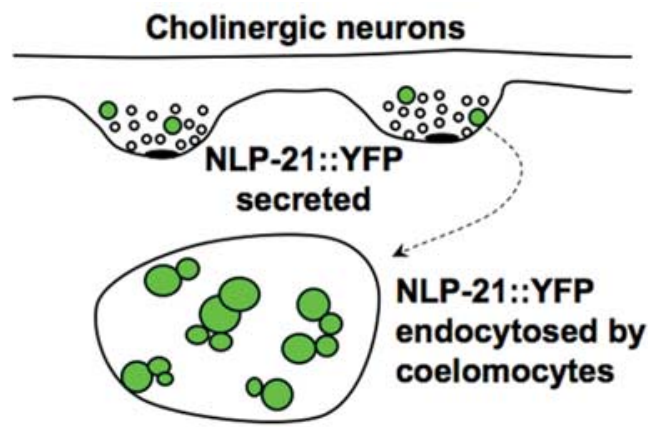

\section{B Wild-type}
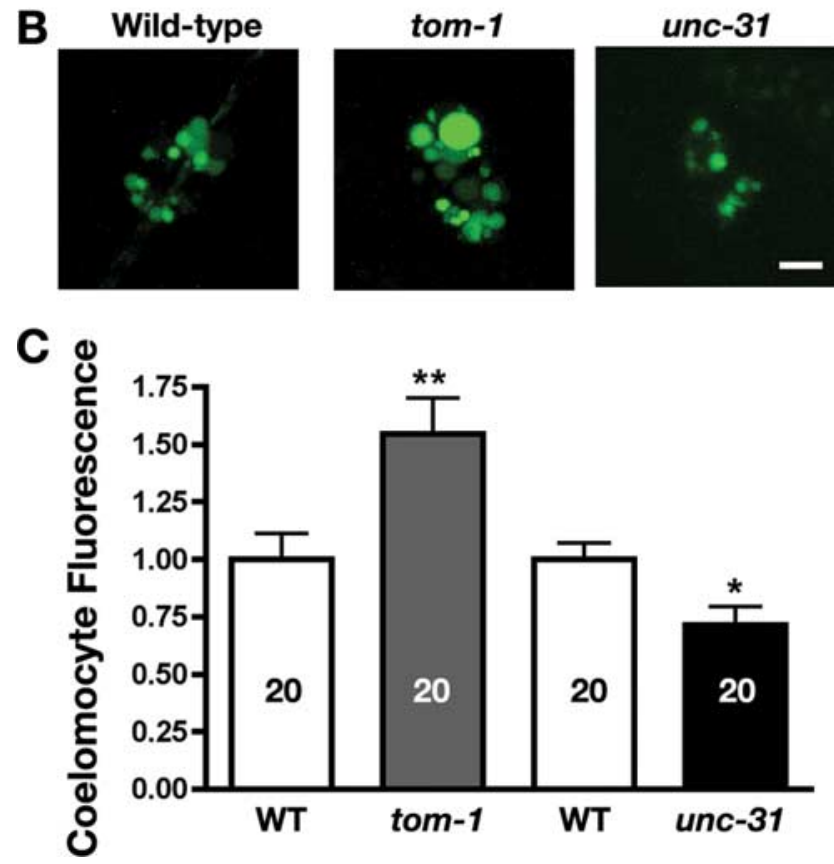

Figure 2. tom-1 and unc-31 mutants exhibit altered peptide release. $\boldsymbol{A}$, Schematic of the peptide secretion assay, in which YFP(Venus)-tagged neuropeptide NLP-21 (KP\#1383 punc-129::n/p-21::Venus), once released from cholinergic neuron DCVs, is taken up by scavenger cells known as coelomocytes. $\boldsymbol{B}$, Representative images of a single coelomocyte from wildtype, tom-1(ok285), and unc-31(e928) young adult worms showing multiple lysosomes containing NLP-21::YFP previously secreted from cholinergic neurons. Scale bar, $10 \mu \mathrm{m}$. C, Average fluorescent intensity values [normalized to wild type (WT) matched for image acquisition parameters] measured from the posterior ventral coelomocyte of wild-type, tom-1(ok285), and unc-31(e928) worms expressing NLP-21:YFP in cholinergic neurons. The significance values (asterisks) above the bars are relative to the wild type, and numbers in bars represent the sample size (n).

in the present study (Fig. $2 B, C$ ). We would therefore expect impaired peptidergic release in $u n c-31$ mutants to result in DCV accumulations in affected synaptic terminals. To test this prediction, we prepared $u n c-31$ mutant worms for ultrastructural anal- ysis. Compared with wild-type synapses, unc-31(e928) mutants exhibited an approximate threefold increase in the number of synaptic DCVs (Fig. 4A, $B$; Table 1). The accumulation of DCVs in conjunction with reduced NLP-21 release in C. elegans unc-31 mutants (Sieburth et al., 2006) supports previous studies implicating CAPS in the promotion of DCV release.

\section{Genetic interactions between tom-1 and unc-31 mutants}

The data presented above indicate that TOM-1 and UNC-31 have opposing effects on DCV exocytosis. We therefore asked whether tom-1 mutants could suppress the peptidergic signaling defects caused by loss of UNC-31 function, by comparing the phenotypes of unc-31 single mutants with those of tom-1;unc-31 double mutants.

unc-31 mutants have pronounced locomotory defects (Charlie et al., 2006), with basal locomotion rates reduced to $20 \%$ of the wild type (unc-31, $3.8 \pm 0.5$ body bends/min, $n=10$, vs wild type, $18.95 \pm 0.4$ body bends/min, $n=10$ ) and head tap responses reduced to $31 \%$ of wild type (unc-31, $0.3 \pm 0.05$ body bends/s, $n=7$, vs wild type, $0.97 \pm 0.078$ body bends/s, $n=10$ ) (Fig. $3 E, F)$. We found that these behavioral defects were significantly improved in tom-1;unc-31 double mutants (locomotion, $13.9 \pm$ 3.8 body bends/min, $n=10$; head tap responses, $0.64 \pm 0.075$ body bends/s, $n=10$ ), although they were still impaired relative to wild-type worms (Fig. $3 E, F$ ). These results suggest that tom-1 mutants can partially suppress the $u n c-31$ mutant behavioral abnormalities.

The locomotory defects in $u n c-31$ mutants have been attributed to loss of downstream signaling in the $\mathrm{G} \alpha$ s pathway acting predominantly in cholinergic neurons on unidentified effectors (Charlie et al., 2006). Given that unc-31 mutants have reduced peptidergic secretion, it is therefore postulated that their paralysis results from reduced activation of peptidergic receptors coupled to $\mathrm{G} \alpha_{\mathrm{S}}$ (Charlie et al., 2006). Consistent with this model, mutants affecting proneuropeptide processing enzymes (egl-3 and egl-21), neuropeptide synthesis (ins-22, ins-31, flp-1, and nlp12), and neuropeptide reception ( $f$ sh $r-1)$ all exhibit impaired cholinergic signaling in whole-worm pharmacological assays, similar to that of unc-31 mutants (Jacob and Kaplan, 2003; Sieburth et al., 2005; Charlie et al., 2006). Based on these observations, we examined the evoked responses of unc-31 mutant NMJs, which are mostly attributable to ACh release (Touroutine et al., 2005). unc-31 mutants exhibited a significant reduction in evoked response amplitudes compared with the wild type (Fig. 4D,E; Table 1), whereas the endogenous mini rates were not significantly affected (unc$31,75.2 .6 \pm 12.2$ events/s, $n=10$, vs wild type, $94.7 \pm 6.9$. events/s, $n=49)$. To test whether the behavioral rescue observed in tom-1;unc-31 double mutants was accompanied by improved transmission at the NMJ, we recorded evoked responses from these worms. Consistent with the behavioral observations, the 
evoked responses were significantly improved in tom-1;unc-31 double mutants compared with unc-31 alone, although they were not restored to either wild-type levels or to the extent observed in tom-1 single mutants (Fig. 4D,E; Table 1).

To test whether the reduced evoked responses observed in unc-31 mutants were caused by defects in presynaptic release or reduced postsynaptic reception, we measured mini amplitudes. The average mini amplitude of unc-31 mutants was not significantly different from wild type (unc$31,26.6 \pm 1.85 \mathrm{pA}, n=10$, vs wild type, $24.5 \pm 0.94 \mathrm{pA}, n=49)$, indicating that the NMJ transmission defect in unc-31 mutants is presynaptic. This conclusion is supported by previous observations that the behavioral responses of $u n c-31$ mutant worms to the cholinergic muscle receptor agonist levamisole are completely normal (Charlie et al., 2006). The mini amplitudes of tom-1;unc-31 double mutants were also unaffected $(27.1 \pm 1.97 \mathrm{pA} ; n=16)$, indicating that the suppression of $u n c-31$ evoked synaptic defects by the tom-1 mutant was not attributable to enhanced postsynaptic reception.

To better understand how tom-1 mutants impact synaptic function in $u n c-31$ mutants, we compared the synaptic ultrastructure of unc-31 and tom-1;unc-31 NMJs. unc-31 mutants did not accumulate SV s but showed a significant reduction in the absolute number of plasma membrane contacting vesicles (Fig. 4C). Under these rapid freeze fixation conditions, we have previously shown that contacting vesicles essentially reflect the primed vesicle pool at C. elegans NMJs (Weimer et al., 2006). Thus, these data suggest that unc-31 mutants may have fewer primed vesicles, which could explain the reduced evoked response. However, measurement of the primed vesicle pool using hyperosmotic saline failed to detect a significant difference between $u n c-31$ mutants and wildtype synapses (unc-31, $12.9 \pm 3.4 \mathrm{pC}, n=$ 9, vs wild type, $15.9 \pm 2.7 \mathrm{pC}, n=9$ ) (Fig. $4 F, G)$. Furthermore, tom-1;unc-31 mutants did not significantly rescue the contacting vesicle pool (Fig. 4C), suggesting that suppression of the $u n c-31$ evoked synaptic defect by tom-1 is not attributable to recovery of SV priming. We therefore asked whether the suppression of $u n c-31$ by tom- 1 was associated with restored peptidergic transmission by examining the number of DCVs in the tom-1;unc-31 double mutants. The average DCV count was significantly reduced in tom-1;unc-31 compared with $u n c-31$ single mutants but was not depleted to the same extent as
A
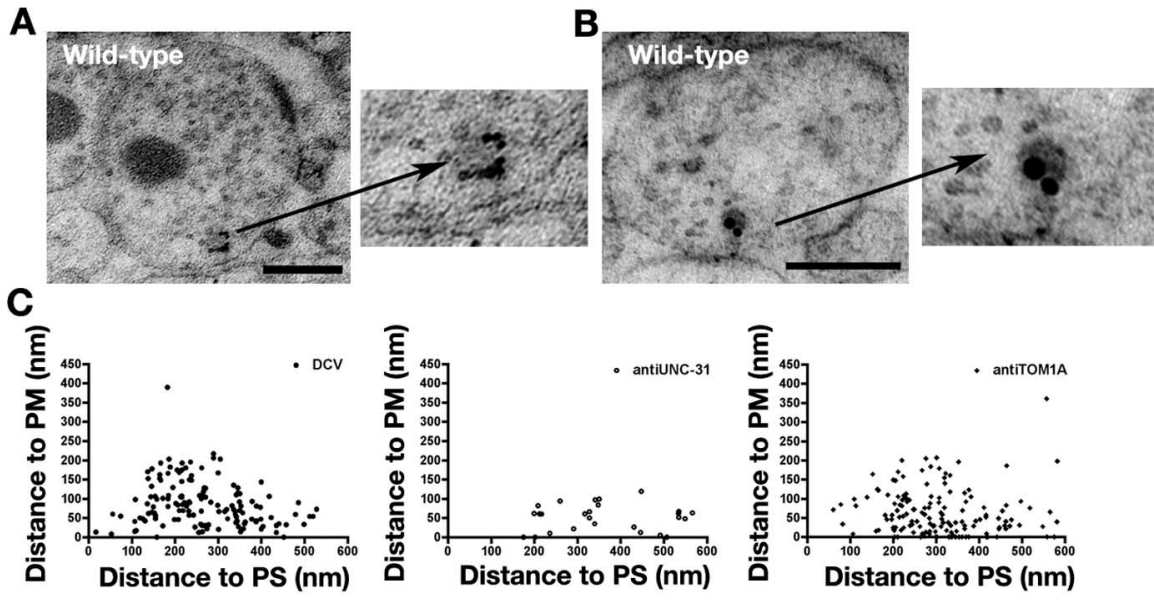

D
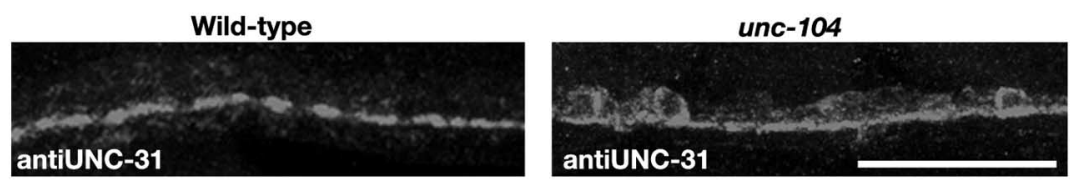

E
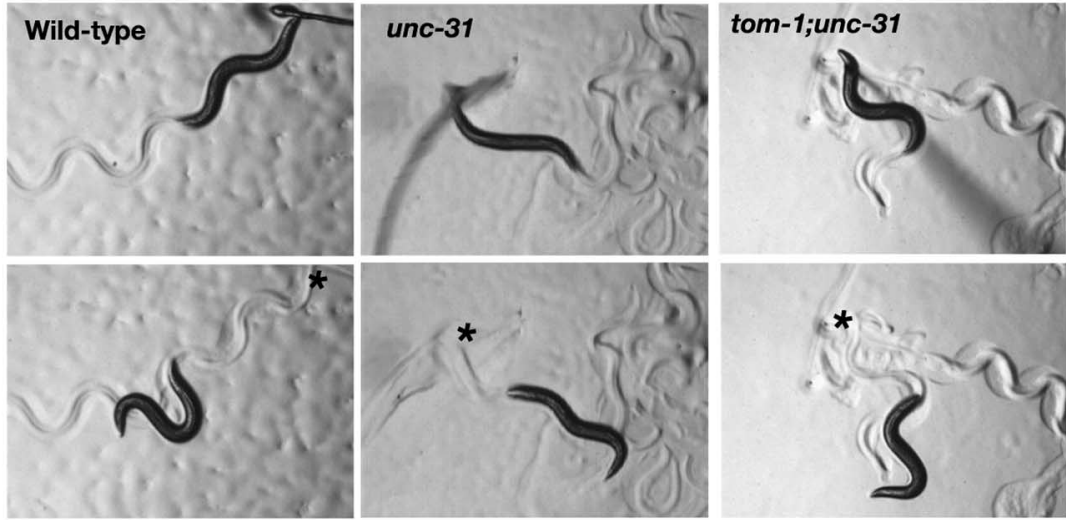

$\mathbf{F}$
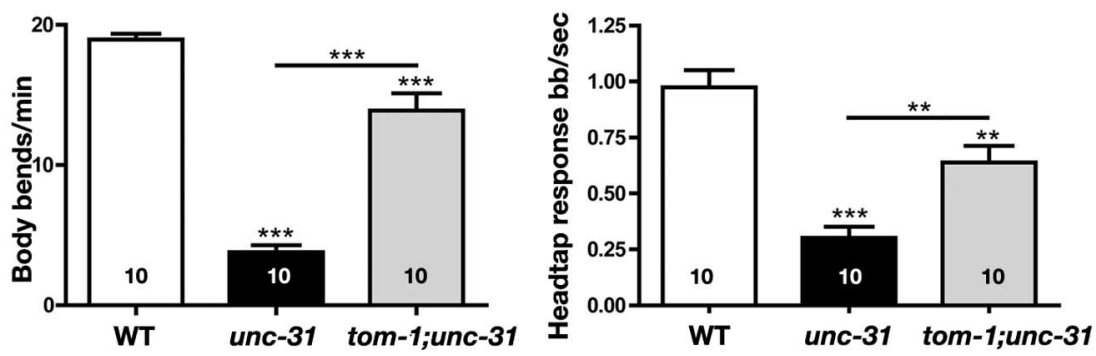

Figure 3. tom-1 mutants suppress the behavioral deficits resulting from loss of DCV-associated UNC-31(CAPS). $\boldsymbol{A}$, Representative micrograph showing immunogold labeling of UNC-31 in an NMJ profile from a wild-type worm. The inset shows an enlargement of clustered immunogold particles surrounding a DCV. B, Representative micrograph showing immunogold labeling of TOM-1A in an NMJ profile from a wild-type worm. The inset shows an enlargement of clustered immunogold particles surrounding a DCV.C, The distribution of DCVs (left), anti-UNC-31 immunogold beads (middle), and anti-Tom1A immunogold beads (right) graphed as scatter plots. Each point represents the measured linear distance of a DCV or bead to the presynaptic specialization and the perpendicular linear distance to the closest plasma membrane (PM). D, UNC-31 immunofluorescent staining of the ventral nerve cord in wild-type punctate staining representing synapses and unc-104 (kinesin motor protein) mutants-neuronal cell bodies show mislocalized UNC-31 immunostaining. $\boldsymbol{E}$, Representative frames from movies used to measure response velocities to heads taps of young adult wild-type, unc-31, and tom-1;unc-31 double mutants. The top panels show the eyelash tapping the head of the worm. The bottom panels show the extent of the reversal response after $3.6 \mathrm{~s}$, at which time average wild-type responses are complete. An asterisk in the bottom panels marks the starting position of the head when the tap was applied. $\boldsymbol{F}$, Behavioral parameters measured for the three genotypes. Left, Average number of body bends during basal locomotion measured for $1 \mathrm{~min}$. Right, Average head tap response expressed as body bends per second (bb/sec) to complete the reversal response. Scale bars: $\boldsymbol{A}, \boldsymbol{B}, 200 \mathrm{~nm} ; \boldsymbol{D}, 20 \mu \mathrm{m}$. PS, Presynaptic specialization; WT, wild type. Significance relative to wild type is displayed directly above the bars (asterisks); all other statistically significant pairwise values are displayed above. Numbers in the bars represent sample size $(n)$. 

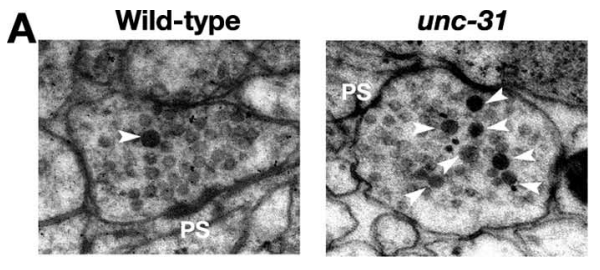

B
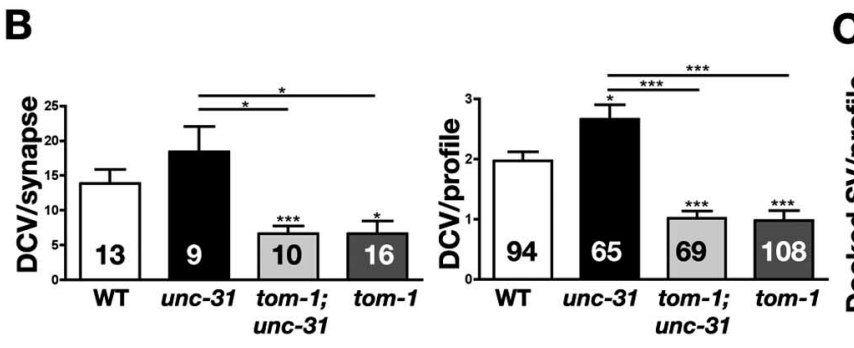

C
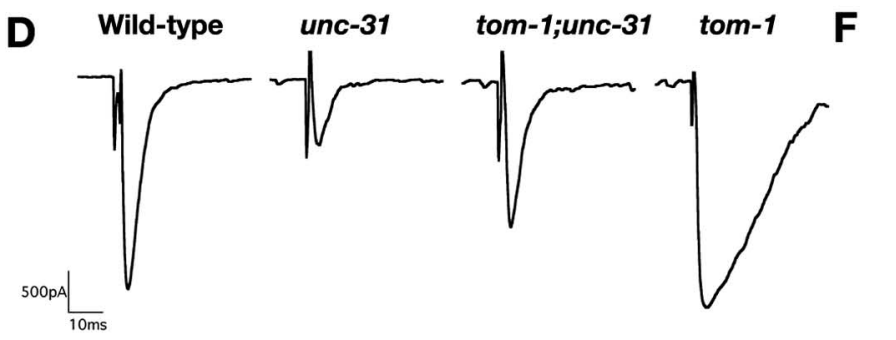

E

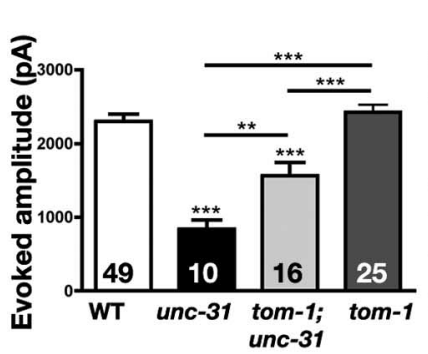

unc-31

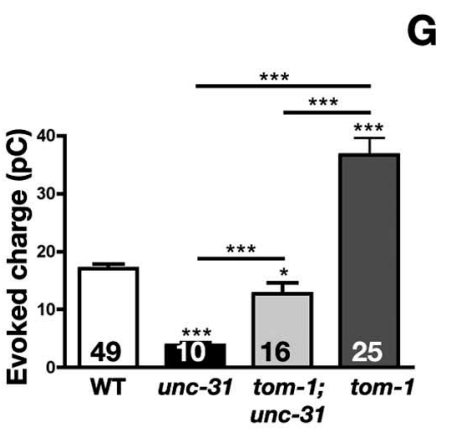

G

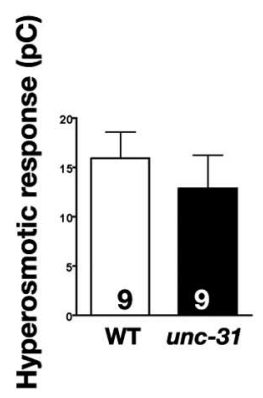

Figure 4. tom-1 mutants suppress the evoked deficit and DCV accumulation of unc-31 mutants. $\boldsymbol{A}$, Representative micrographs of NMJ profiles for wild-type (WT), unc-31, tom-1;unc-31, and tom-1 mutant synapses. The presynaptic density-presynaptic specialization (PS) and DCVs (arrowheads) are indicated. Scale bar, $200 \mathrm{~nm}$. B, Graphs of average DCV per synapse (left) and average DCV per profile (right) measured for the four genotypes. $C$, Graph of the average number of plasma membrane contacting (docked) SVs for wild-type, unc-31, and tom-1; unc-31 mutant synaptic profiles $\boldsymbol{D}$, Representative evoked postsynaptic currents from ventral NMJs of wild-type, unc-31, tom-1;unc-31, and tom-1 mutants, in response to a 2 ms depolarization of the ventral nerve cord. $\boldsymbol{E}$, Plots of the average evoked amplitude (left) and total charge transfer (right) for the three genotypes. $\boldsymbol{F}$, Representative hyperosmotic responses from ventral NMJs of wild-type and unc-31 mutants. G, Plots of the average hyperosmotic responses for wild-type and unc-31 mutants (expressed as total charge transfer for synaptic events in response to a 1.5 s sucrose application). In $\boldsymbol{B}, \boldsymbol{C}, \boldsymbol{E}$, and $\mathbf{G}$, significance relative to wild-type is displayed directly above the bars (asterisks); all other statistically significant pairwise values are displayed above. Numbers in the bars represent sample size $(n)$.

tom-1 single mutants (Fig. $4 A, B$; Table 1). Together, these results indicate that tom -1 and $u n c-31$ genetically interact and that loss of TOM-1 can partially restore peptide release in the absence of UNC-31, leading to improvements in locomotion and synaptic function.

The regulation of DCVs by TOM-1 is not caused by altered synaptic activity

DCV release is thought to require higher levels of neuronal excitability than SV release (Bruns and Jahn, 1995), suggesting that DCV release is likely to be impaired in mutants exhibiting reduced synaptic activity. Both unc-31 mutants and TOM-

\section{Discussion}

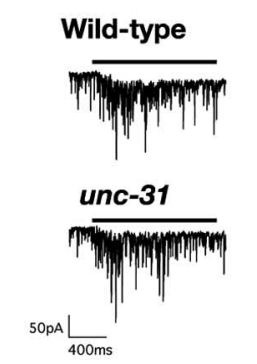

1A( jaIs1052)-overexpressing synapses exhibit similar decreases in evoked synaptic responses (Fig. 4D,E; Table 1) (Gracheva et al., 2006). Therefore, it is possible that the accumulation of DCVs in unc-31 and TOM-1A(jaIs1052), are indirect consequences of reduced neuronal excitability, rather than direct effects on the DCV release machinery. To test this possibility, we analyzed DCV abundance in the synapses of $u n c-10$ (Rim) mutants, which exhibit a similar decrease in evoked release (Fig. $5 A, B$ ) and a more severe defect in endogenous synaptic activity $(17.7 \pm 4.25$ events/s; $n=5)$ relative to $u n c-31$ (75.2 \pm 12.2 events/s; $n=10)$ and TOM1A(jaIs1052) (Koushika et al., 2001; Gracheva et al., 2006). UNC-10(Rim) is a component of the presynaptic dense projection, implicated in the targeting and priming of SVs but not DCVs (Koushika et al., 2001; Weimer et al., 2006). We found that the DCVs in unc-10 mutant synapses were significantly less abundant than both unc-31 and TOM-1A( jaIs1052) (Fig. $5 C, D$ ). These data indicate that the DCV accumulations observed in unc-31 mutants and TOM-1A-overexpressing neurons are not simply a reflection of reduced SV release.

Because tom-1 loss-of-function mutants have previously been shown to exhibit enhanced evoked release (Gracheva et al., 2006; McEwen et al., 2006), we also questioned whether the increased release of DCVs in tom-1 mutants was the result of increased neuronal excitability rather than direct promotion of DCV release. To address this question, we generated tom-1;unc-10(md1117) double mutants. The evoked and endogenous (39.4 \pm 5.03 events/s; $n=6)$ synaptic responses of tom-1;unc-10 double mutants were partially restored compared with unc-10 alone but remained much lower than those observed in either wildtype or tom-1 single mutants (Fig. $5 A, B)$. These double mutants therefore provided an opportunity to examine the effects of the tom-1 mutant in the absence of enhanced synaptic activity. When we examined DCV numbers in tom-1;unc-10 double mutants, we found they were reduced to the same extent as tom-1 single mutants (Fig. $5 C, D$; Table 1). These data indicate that the ability of TOM-1 to regulate DCV release is independent of its actions on SV release and argue for a direct role of TOM-1 in the regulation of peptidergic transmission.

We investigated the role of tomosyn in neuronal DCV exocytosis through the analysis of tom-1 loss-of-function mutants in the genetically tractable model organism $C$. elegans. We found that tom-1 mutant synapses possess fewer DCVs, whereas TOM-1 
overexpression results in DCV accumulation, suggesting TOM-1 negatively regulates DCV release. We confirmed this hypothesis by demonstrating that YFPtagged NLP-21 secretion from cholinergic NMJs was increased in tom-1 mutants relative to the wild type. This conclusion is in agreement with tomosyn overexpression studies in cultured endocrine cells in which DCV exocytosis was shown to be inhibited (Fujita et al., 1998; Hatsuzawa et al., 2003; Yizhar et al., 2004) and is also supported by one of two tomosyn RNAi studies showing enhanced release from pancreatic $\beta$-cells (Zhang et al., 2006). We found no evidence for a permissive role of TOM-1, as proposed in other studies of tomosyn function (Baba et al., 2005; Cheviet et al., 2006).

We have previously shown that C. elegans tom-1 mutants have enhanced SV release, yet the number of SVs in tom-1 mutant synapses was normal (Gracheva et al., 2006). In contrast, here we observed a $50 \%$ reduction in the number of DCVs in the same tom-1 mutant synapses. We attribute the specific depletion of DCVs to the inability of tom-1 mutant neurons to replenish the synaptic DCV pool because of the requirement for de novo synthesis, UNC-104 (kinesin)-dependent transport (Zahn et al., 2004), and/or local capture of DCVs at synaptic terminals (Shakiryanova et al., 2006). In contrast, SVs can be rapidly recycled, allowing the SV pool to keep pace with the increased exocytosis observed in tom-1 mutants. Consistent with the reduction in DCV numbers in tom-1 mutants, TOM-1 overexpression caused DCVs to accumulate in synapses. However, although tomosyn overexpression in PC12 cells also inhibits DCV release, DCV abundance was not found to be affected (Yizhar et al., 2004). We speculate that given the scarcity of DCVs in worm NMJs compared with PC12 cells, changes in DCV density may be more readily detectable at NMJs.

Although the molecular mechanism by which tomosyn restricts DCV fusion has yet to be fully elucidated, biochemical (Fujita et al., 1998; Hatsuzawa et al., 2003) and crystallographic (Pobbati et al., 2004; Hattendorf et al., 2007) data provide evidence for a model in which competition between tomosyn and synaptobrevin for binding to the plasma membrane SNAREs syntaxin and SNAP-25 limits the assembly of synaptobrevincontaining SNARE complexes required for vesicle fusion. We have previously shown that C. elegans TOM-1 is capable of forming an SDS-sensitive complex with syntaxin and SNAP-25 (Gracheva et al., 2006) and that the priming of SVs in C. elegans is inhibited by TOM-1 (Gracheva et al., 2006; McEwen et al., 2006). The present study establishes that TOM-1 also negatively regulates DCV release in vivo, implying that tomosyn may inhibit the priming of both vesicle classes through a common mode of action involving the SNARE proteins.

To ascertain whether TOM-1 acts in the DCV priming pathway, we examined the functional relationship between TOM-1 and the presumptive DCV priming factor, CAPS. Although the mechanism by which CAPS promotes DCV release has yet to be fully elucidated, evidence indicates that CAPS functions at a late stage of DCV secretion (Hay and Martin, 1992; Walent et al., 1992; Ann et al., 1997). Furthermore, CAPS has a conserved Munc13 homology domain that in Munc13/UNC-13 proteins is required to promote $\mathrm{SV}$ priming through an interaction with syntaxin (Basu et al., 2005; Madison et al., 2005; Stevens et al., 2005). Whether CAPS interacts in a similar manner with syntaxin and whether CAPS promotes SNARE complex assembly has yet to be determined.

To study the potential interaction between TOM-1 and $C$. elegans CAPS (UNC-31) in peptide release, we first characterized the ultrastructural phenotype of unc-31 mutants. Loss of UNC-31 resulted in a threefold accumulation of synaptic DCVs indicative of a peptidergic release defect. This correlated well with previous reports of reduced neuropeptide secretion in unc-31 mutants (Sieburth et al., 2006; Speese et al., 2007). Drosophila CAPS mutants exhibit a similar accumulation of DCVs at NMJs (Renden et al., 2001). These observations support a role for UNC-31(CAPS) in the promotion of DCV exocytosis, although our data do not rule out the possibility of additional roles for CAPS in peptide storage (Speidel et al., 2005) or the regulation of 
fusion kinetics (Rupnik et al., 2000) proposed in other CAPS studies. Examination of tom-1;unc-31 mutants indicates that tom-1 mutants partially suppress the DCV accumulation caused by loss of UNC-31. On the basis of these genetic interactions, we propose that TOM-1 restricts the UNC-31-dependent fusion of DCVs possibly through inhibitory interactions with syntaxin and SNAP-25.

In this study, we also demonstrated that $u n c-31$ mutants have impaired evoked synaptic transmission. A similar reduction in synaptic transmission has been observed in Drosophila CAPS mutants, and yet in both organisms, endogenous mini frequencies were not significantly affected (Renden et al., 2001). Precisely how CAPS specifically regulates evoked SV release remains to be elucidated. We know that loss-of-function mutants in the $\mathrm{G} \alpha_{\mathrm{S}}$ pathway exhibit profound locomotory defects similar to those of unc-31 mutants. Furthermore, C. elegans unc-31 mutants can be rescued by activation of the $\mathrm{G} \alpha_{\mathrm{S}}$ pathway, but the downstream effectors remain to be identified. Based on these observations, two alternative models can be proposed for UNC-31 regulation of SV exocytosis: (1) UNC-31-dependent peptide release may modulate synaptic transmission by activating peptidergic receptors coupled to $\mathrm{G} \alpha_{\mathrm{S}}$, and/or (2) UNC-31 may directly regulate evoked SV exocytosis. The modest reduction we observed in contacting vesicles in $u n c-31$ mutants was not accompanied by a detectable decrease in the size of hyperosmotic responses, suggesting that if UNC-31 regulates SV priming, the effect is marginal. These data do rule out the possibility that UNC-31 directly regulates a step downstream of SV priming. However, a recent study found no discernable changes in SV recycling in cultured neurons from C. elegans unc-31 mutants measured by FM4-64 dye uptake (Speese et al., 2007), whereas, in the same study, peptide release from cultured neurons was dramatically reduced in unc-31 mutants. Together, these data argue for an indirect role of UNC-31 in synaptic transmission, through the release of peptides acting via the $\mathrm{G} \alpha_{\mathrm{S}}$ pathway. Because UNC-31 is pan-neuronal, the origin of the peptides regulating motor neuron output remains to be fully elucidated. However, behavioral rescue of unc-31 mutants by expression of UNC-31 or activation of the $\mathrm{G} \alpha_{\mathrm{S}}$ pathway specifically in cholinergic neurons suggests that the cholinergic motor neurons play a prominent role in this regulatory pathway. Interestingly, tom-1 mutants show only partial rescue of the evoked synaptic defect of unc-31 mutants. This suggests that a fully functional UNC-31 regulatory pathway is required for tom-1 mutants to exhibit enhanced release relative to the wild type.

\section{References}

Ann K, Kowalchyk JA, Loyet KM, Martin TF (1997) Novel Ca2+-binding protein (CAPS) related to UNC-31 required for Ca2+-activated exocytosis. J Biol Chem 272:19637-19640.

Ashery U, Varoqueaux F, Voets T, Betz A, Thakur P, Koch H, Neher E, Brose N, Rettig J (2000) Munc13-1 acts as a priming factor for large densecore vesicles in bovine chromaffin cells. EMBO J 19:3586-3596.

Baba T, Sakisaka T, Mochida S, Takai Y (2005) PKA-catalyzed phosphorylation of tomosyn and its implication in Ca2+-dependent exocytosis of neurotransmitter. J Cell Biol 170:1113-1125.

Basu J, Shen N, Dulubova I, Lu J, Guan R, Guryev O, Grishin NV, Rosenmund C, Rizo J (2005) A minimal domain responsible for Munc13 activity. Nat Struct Mol Biol 12:1017-1018.

Berwin B, Floor E, Martin TF (1998) CAPS (mammalian UNC-31) protein localizes to membranes involved in dense-core vesicle exocytosis. Neuron 21:137-145.

Broadie K, Prokop A, Bellen HJ, O’Kane CJ, Schulze KL, Sweeney ST (1995) Syntaxin and synaptobrevin function downstream of vesicle docking in Drosophila. Neuron 15:663-673.
Bruns D, Jahn R (1995) Real-time measurement of transmitter release from single synaptic vesicles. Nature 377:62-65.

Charlie NK, Schade MA, Thomure AM, Miller KG (2006) Presynaptic UNC-31 (CAPS) is required to activate the G\{alpha\}s pathway of the Caenorhabditis elegans synaptic signaling network. Genetics 172:943-961.

Chen YA, Scheller RH (2001) SNARE-mediated membrane fusion. Nat Rev Mol Cell Biol 2:98-106.

Cheviet S, Bezzi P, Ivarsson R, Renstrom E, Viertl D, Kasas S, Catsicas S, Regazzi R (2006) Tomosyn-1 is involved in a post-docking event required for pancreatic beta-cell exocytosis. J Cell Sci 119:2912-2920.

Dybbs M, Ngai J, Kaplan JM (2005) Using microarrays to facilitate positional cloning: identification of tomosyn as an inhibitor of neurosecretion. PLoS Genet 1:6-16.

Fujita Y, Shirataki H, Sakisaka T, Asakura T, Ohya T, Kotani H, Yokoyama S, Nishioka H, Matsuura Y, Mizoguchi A, Scheller RH, Takai Y (1998) Tomosyn: a syntaxin-1-binding protein that forms a novel complex in the neurotransmitter release process. Neuron 20:905-915.

Gracheva EO, Burdina AO, Holgado AM, Berthelot-Grosjean M, Ackley BD, Hadwiger G, Nonet ML, Weimer RM, Richmond JE (2006) Tomosyn inhibits synaptic vesicle priming in Caenorhabditis elegans. PLoS Biol $4: \mathrm{e} 261$.

Grishanin RN, Klenchin VA, Loyet KM, Kowalchyk JA, Ann K, Martin TF (2002) Membrane association domains in Ca2+-dependent activator protein for secretion mediate plasma membrane and dense-core vesicle binding required for $\mathrm{Ca} 2+$-dependent exocytosis. J Biol Chem 277:22025-22034.

Hanson PI, Heuser JE, Jahn R (1997) Neurotransmitter release-four years of SNARE complexes. Curr Opin Neurobiol 7:310-315.

Hatsuzawa K, Lang T, Fasshauer D, Bruns D, Jahn R (2003) The R-SNARE motif of tomosyn forms SNARE core complexes with syntaxin 1 and SNAP-25 and down-regulates exocytosis. J Biol Chem 278:31159-31166.

Hattendorf DA, Andreeva A, Gangar A, Brennwald PJ, Weis WI (2007) Structure of the yeast polarity protein Sro7 reveals a SNARE regulatory mechanism. Nature 446:567-571.

Hay JC, Martin TF (1992) Resolution of regulated secretion into sequential MgATP-dependent and calcium-dependent stages mediated by distinct cytosolic proteins. J Cell Biol 119:139-151.

Jacob TC, Kaplan JM (2003) The EGL-21 carboxypeptidase E facilitates acetylcholine release at Caenorhabditis elegans neuromuscular junctions. J Neurosci 23:2122-2130.

Koushika SP, Richmond JE, Hadwiger G, Weimer RM, Jorgensen EM, Nonet ML (2001) A post-docking role for active zone protein Rim. Nat Neurosci 4:997-1005.

Lonart G, Sudhof TC (2000) Assembly of SNARE core complexes prior to neurotransmitter release sets the readily releasable pool of synaptic vesicles. J Biol Chem 275:27703-27707.

Madison JM, Nurrish S, Kaplan JM (2005) UNC-13 interaction with syntaxin is required for synaptic transmission. Curr Biol 15:2236-2242.

McEwen JM, Madison JM, Dybbs M, Kaplan JM (2006) Antagonistic regulation of synaptic vesicle priming by Tomosyn and UNC-13. Neuron 51:303-315.

Pobbati A, Razeto A, Boddener M, Becker S, Fasshauer D (2004) Structural basis for the inhibitory role of tomosyn in exocytosis. J Biol Chem 279:47192-47200.

Renden R, Berwin B, Davis W, Ann K, Chin CT, Kreber R, Ganetzky B, Martin TF, Broadie K (2001) Drosophila CAPS is an essential gene that regulates dense-core vesicle release and synaptic vesicle fusion. Neuron 31:421-437.

Richmond JE, Davis WS, Jorgensen EM (1999) UNC-13 is required for synaptic vesicle fusion in C. elegans. Nat Neurosci 2:959-964.

Richmond JE, Weimer RM, Jorgensen EM (2001) An open form of syntaxin bypasses the requirement for UNC-13 in vesicle priming. Nature 412:338-341.

Rostaing P, Weimer RM, Jorgensen EM, Triller A, Bessereau JL (2004) Preservation of immunoreactivity and fine structure of adult C. elegans tissues using high-pressure freezing. J Histochem Cytochem 52:1-12.

Rupnik M, Kreft M, Sikdar SK, Grilc S, Romih R, Zupancic G, Martin TF, Zorec R (2000) Rapid regulated dense-core vesicle exocytosis requires the CAPS protein. Proc Natl Acad Sci USA 97:5627-5632.

Shakiryanova D, Tully A, Levitan ES (2006) Activity-dependent synaptic capture of transiting peptidergic vesicles. Nat Neurosci 9:896-900.

Sieburth D, Ch'ng Q, Dybbs M, Tavazoie M, Kennedy S, Wang D, Dupuy D, 
Rual JF, Hill DE, Vidal M, Ruvkun G, Kaplan JM (2005) Systematic analysis of genes required for synapse structure and function. Nature 436:510-517.

Sieburth D, Madison JM, Kaplan JM (2006) PKC-1 regulates secretion of neuropeptides. Nat Neurosci 10:49-57.

Sollner T, Whiteheart SW, Brunner M, Erdjument-Bromage H, Geromanos S, Tempst P, Rothman JE (1993) SNAP receptors implicated in vesicle targeting and fusion. Nature 362:318-324.

Speese S, Petrie M, Schuske K, Ailion M, Ann K, Iwasaki K, Jorgensen EM, Martin TF (2007) UNC-31 (CAPS) is required for dense-core vesicle but not synaptic vesicle exocytosis in Caenorhabditis elegans. J Neurosci 27:6150-6162.

Speidel D, Bruederle CE, Enk C, Voets T, Varoqueaux F, Reim K, Becherer U, Fornai F, Ruggieri S, Holighaus Y, Weihe E, Bruns D, Brose N, Rettig J (2005) CAPS1 regulates catecholamine loading of large dense-core vesicles. Neuron 46:75-88.

Stevens DR, Wu ZX, Matti U, Junge HJ, Schirra C, Becherer U, Wojcik SM, Brose N, Rettig J (2005) Identification of the minimal protein domain required for priming activity of Munc13-1. Curr Biol 15:2243-2248.

Touroutine D, Fox RM, Von Stetina SE, Burdina A, Miller III DM, Richmond JE (2005) acr-16 encodes an essential subunit of the levamisole-resistant nicotinic receptor at the Caenorhabditis elegans neuromuscular junction. J Biol Chem 280:27013-27021.

Walent JH, Porter BW, Martin TF (1992) A novel $145 \mathrm{kd}$ brain cytosolic protein reconstitutes $\mathrm{Ca}(2+)$-regulated secretion in permeable neuroendocrine cells. Cell 70:765-775.

Weimer RM, Richmond JE (2005) Synaptic vesicle docking: a putative role for the Munc18/Sec1 protein family. Curr Top Dev Biol 65:83-113.

Weimer RM, Gracheva EO, Meyrignac O, Miller KG, Richmond JE, Bessereau JL (2006) UNC-13 and UNC-10/rim localize synaptic vesicles to specific membrane domains. J Neurosci 26:8040-8047.

Yizhar O, Matti U, Melamed R, Hagalili Y, Bruns D, Rettig J, Ashery U (2004) Tomosyn inhibits priming of large dense-core vesicles in a calciumdependent manner. Proc Natl Acad Sci USA 101:2578-2583.

Yokoyama S, Shirataki H, Sakisaka T, Takai Y (1999) Three splicing variants of tomosyn and identification of their syntaxin-binding region. Biochem Biophys Res Commun 256:218-222.

Zahn TR, Angleson JK, MacMorris MA, Domke E, Hutton JF, Schwartz C, Hutton JC (2004) Dense core vesicle dynamics in Caenorhabditis elegans neurons and the role of kinesin UNC-104. Traffic 5:544-559.

Zhang W, Lilja L, Mandic SA, Gromada J, Smidt K, Janson J, Takai Y, Bark C, Berggren PO, Meister B (2006) Tomosyn is expressed in beta-cells and negatively regulates insulin exocytosis. Diabetes 55:574-581. 